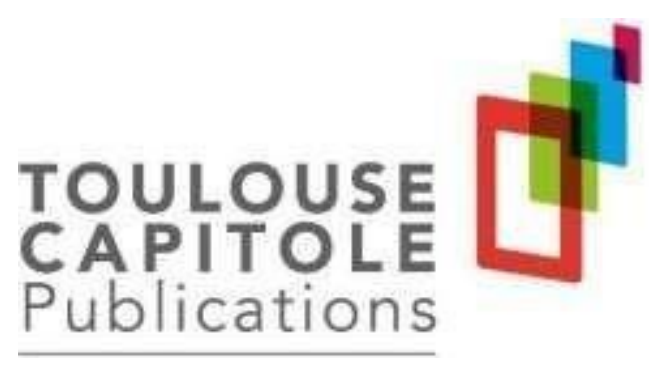

« Toulouse Capitole Publications » est l'archive institutionnelle de l'Université Toulouse 1 Capitole.

Le droit de l'Union européenne et la lutte contre la fraude et l'évasion fiscales : État des lieux

RAIMBAULT DE FONTAINE SOPHIE

Référence de publication : RAIMBAULT DE FonTAINE (S.), « Le droit de l'Union européenne et la lutte contre la fraude et l'évasion fiscales : État des lieux », Gestion et finances publiques, 2020, (nº 4$)$. p. 115122. 


\title{
Le droit de l'Union européenne et la lutte contre la fraude et l'évasion fiscales : État des lieux
}

\begin{abstract}
La lutte contre la fraude et l'évasion fiscale est devenu un élément important du programme de la Commission. Le droit de l'Union a pour premier objectif d'améliorer les outils nationaux en favorisant la coopération et en généralisant les dispositifs anti-abus. Les textes et la jurisprudence imposent aux États une obligation de lutte contre les abus des normes européennes et, de plus en plus, un devoir de combattre la fraude à leur propre législation.
\end{abstract}

Initialement concentrée sur la construction du marché unique par l'élimination des obstacles fiscaux aux échanges, l'action de l'Union européenne semble avoir été, pendant le mandat de la Commission Juncker et au nom de la justice fiscale, toute entière tournée vers la lutte contre la fraude et l'évasion fiscales.

L'attention portée aux pratiques d'évitement de l'impôt n'est pas nouvelle et l'on sait que la digitalisation de l'économie, couplée aux libertés de circulation, facilite ce type de comportements. Pour autant, les conditions de la nomination de la Commission Junker en plein scandale des Luxleaks ne sont sans doute pas étrangères à l'intensification de l'action européenne en la matière. À l'aube de son mandat, son Président avait d'ailleurs insisté sur la « nécessité d'intensifier les efforts pour lutter contre l'évasion et la fraude fiscale, afin que chacun apporte sa juste contribution $\gg 1$. Cependant, les succès législatifs obtenus ne sont pas uniquement le fruit du travail de per- suasion de la Commission et doivent être replacés dans le contexte d'une prise de conscience mondiale de l'acuité des problèmes liés aux stratégies de planification fiscale des entreprises. Si la démarche européenne n'est évidemment pas similaire, elle n'en a pas moins été directement influencée par les réflexions menées au sein de l'OCDE dans le cadre inclusif du BEPS.

À cet égard, il est intéressant de remarquer que, si les initiatives récentes ont bien sûr concerné la lutte contre la fraude à la TVA qui reste un sujet majeur au sein de l'Union européenne2, les dernières avancées législatives sont pour la plu- part intervenues dans le domaine de la fiscalité directe des entreprises, domaine dans lequel le rapprochement des législations semblait pour- tant en panne. Par ailleurs, alors que traditionnellement les propositions de la Commission européenne visaient la fraude, notamment la fraude à la TVA de type «carrousel », les directives récentes ciblent l'évasion fiscale3 entendue comme une " optimisation fiscale agressive ", c'est-à-dire l'ensemble des techniques par les- quelles certains contribuables «exploitent les failles juridiques des systèmes fiscaux et les asymétries qui existent entre les règles nationales pour éluder le paiement de leur juste part de l'impôt »4. La lutte concerne donc désormais non seulement des comportements illégaux, mais aussi des montages jusqu'ici licites mais dont l'objectif fiscal déterminant est considéré comme dommageable5.

Au-delà de la production soutenue de normes destinées à améliorer, dans le respect du principe de subsidiarité, les outils nationaux de lutte contre la fraude et l'évasion fiscales, la période récente semble être aussi caractérisée par l'émergence, en droit européen, d'une obligation à la charge des État de lutter contre les comportements frauduleux ou abusifs en matière fiscale.

Jusqu'à récemment en effet, en matière de fiscalité directe, le droit de l'Union se contentait de faciliter les efforts nationaux de lutte contre la fraude tout en encadrant les législations des États membres afin qu'elles ne portent pas at- teinte à l'effectivité du droit primaire ou dérivé de l'Union. La rigueur avec laquelle certains États entendent protéger leurs bases fiscales est en effet de nature à restreindre les libertés de circulation et est, à ce titre, condamnée6. À l'opposé, le relâchement avec lequel d'autres gouvernements luttent contre les comportements d'évitement fiscal est de nature à créer des distorsions de concurrence préjudiciables au fonctionnement du marché intérieur, et participe de la concurrence fiscale dommageable 
que se livrent les États membres. Ainsi par exemple, en matière de TVA, la Commission européenne a clairement pointé ces politiques de dumping procédural en soutenant que la définition d'in- fractions communes pourrait " décourager les contrevenants en puissance d'exercer leurs activités illicites intentionnelles dans des juridictions plus clémentes au sein de l'Union »7. Or, si la dé- finition d'infractions communes dans tous les secteurs de la fiscalité n'est pas encore d'actualité, il n'en reste pas moins que ces dernières années, la Cour de justice, mais également la Commission européenne, se sont de manière très cohérente saisies du droit de l'Union pour s'assurer que les États luttent effectivement contre les comportements frauduleux et abusifs.

Finalement, l'évolution récente du droit de l'Union semble être marquée par deux mouvements complémentaires : d'une part, la reconnaissance par les États membres de l'utilité de légiférer au plan européen pour améliorer leurs outils nationaux de lutte contre l'évitement fiscal (I) ; d'autre part, l'instrumentalisation, par les institutions européennes, du droit de l'Union aux fins de contrôler l'effectivité de l'action des autorités nationales en la matière (II).

\section{I- L'amélioration par le droit de l'Union des outils nationaux de lutte contre les comportements d'évitement fiscal...}

Les directives adoptées récemment ont eu pour objectif premier d'améliorer les outils dont dis- posent les États membres pour lutter contre les comportements frauduleux. Elles le font néanmoins de deux manières différentes : un premier groupe de textes, assez classiquement, élargit les possibilités de coopération entre administrations fiscales étatiques, l'autre, de manière plus novatrice, impose aux États membres d'introduire dans leurs législations nationales un certain nombre de dispositifs anti-abus.

\section{A. La systématisation de l'échange automatique d'informations en matière de fiscalité directe des entre-prises}

La directive 211/16/UE du 15 février 2011 relative à la coopération administrative dans le domaine fiscal (ci-après DAC) a été particulièrement sollicitée : sur les quatre dernières années, elle a en effet été modifiée quatre fois. Il s'est agi non seulement de systématiser l'échange automatique d'informations entre administrations en matière de fiscalité directe - alors que ce mode de coopération était surtout utilisé en matière de TVA - mais également de l'étendre à de nouvelles in- formations, notamment pour le contrôle de l'im- position des bénéfices des entreprises.

Ainsi, suite à la mise en œuvre de la législation américaine FATCA, l'échange automatique d'in- formations antérieurement réservé à certains revenus de l'épargne a été étendu à tous les revenus financiers et soldes de comptes par la directive DAC 2 du 9 décembre 20148. Actant la fin du secret bancaire au sein de 1'UE et alignant les règles européennes sur la norme commune de déclaration telle qu'elle résultait de la convention fiscale adoptée dans le cadre de l'OCDE, ces dispositions obligent désormais les États à transmettre les informations fournies par leurs établissements financiers à l'État de résidence du contribuable titulaire du compte ou des revenus. Les institutions financières déclarantes sont tenues de mettre en œuvre « des procédures de diligence raisonnable » destinées à assurer l'identification du titulaire réel des comptes ou des revenus ainsi que de sa résidence. Les premiers échanges ont eu lieu en 2017 et la directive DAC 59 du 6 décembre 2016 permet aux administrations fiscales d'avoir accès aux données relatives à la lutte contre le blanchiment de capitaux, notamment aux registres centraux des bénéficiaires effectifs, afin qu'elles puissent évaluer et contrôler que les institutions financières déclarantes au titre de la DAC 2 appliquent de manière satisfaisante les procédures de diligence raisonnable.

La directive DAC 310 du 8 décembre 2015 a en- suite étendu l'obligation d'échange automatique aux 
décisions fiscales anticipées en matière transfrontière et aux accords préalables de prix de transfert émis, modifiés ou renouvelés après le 31 décembre 2016. Puis la Directive DAC 411 du 25 mai 2016 a imposé aux États d'échanger les déclarations pays par pays (country by country reports) désormais exigées des entités mères ultimes des groupes d'entreprises opérant sur le territoire européen. Au-delà de l'effet dissuasif à l'égard des États ayant une pratique contestable des rescrits, il s'agit de permettre que soient repérés plus aisément certains cas de planification fiscale agressive, notamment ceux résultant de la manipulation des prix de transfert12. Enfin, la Directive DAC 613 du 25 mai 2018 impose aux États membres de mettre à la charge des inter- médiaires fiscaux, et à défaut des contribuables eux-mêmes, une obligation de déclaration des dispositifs transfrontières porteurs d'un risque potentiel d'évasion fiscale. Les dispositifs soumis à obligation de déclaration sont identifiés par la présence d'un certain nombre de marqueurs décrits par le texte de la directive et les informations collectées sont centralisées dans un répertoire géré par la Commission européenne, répertoire dont l'accès est ouvert aux États membres 14.

Cette rapide présentation atteste des incontestables progrès de la transparence fiscale entre administrations. De manière remarquable, la transparence recherchée ne concerne plus uniquement des informations utiles à la détection de comportements illégaux, mais également celles susceptibles de révéler des pratiques de planification fiscale agressive nécessitant l'adoption de mesures législatives défensives. Pour au- tant, si la Commission et certains États membres ont largement communiqué sur ces mesures, il est trop tôt pour s'enthousiasmer. L'annonce de l'échange automatique sur les comptes financiers a certes eu un effet dissuasif notable sur nombre « d'évadés fiscaux » qui ont régularisé leur situation avant même son entrée en vigueur effective, néanmoins, les effets réels des mesures adoptées sont encore incertains. La lecture du dernier rapport de la Commission européenne est sur ce point particulièrement éclairante : il semble que certains États ne soient pas en mesure de disposer des informations qu'ils doivent transmettre, que les délais de transmission ne soient pas toujours optimums, que d'autres n'utilisent pas les données transmises ou n'aient pas les moyens techniques pour ce faire...15 Plus encore, certains États, Chypre et Malte notamment, ont développé des programmes de résidence contre investissement, dits « visa ou passeports dorés », dont il est établit qu'ils peuvent permettre de contourner les règles d'échanges 16.

Par ailleurs, si l'échange automatique est un outil intéressant, faut-il encore qu'il soit correctement utilisé. Comme le reconnaissait d'ailleurs Pierre Moscovici devant le Parlement européen, la Commission devra " s'assurer que les règles de transparence s'intègrent au travail quotidien des inspecteurs des impôts.... [et] que les États membres y consacrent les moyens nécessaires »17. D’autre part, cet outil ne peut se substituer à une véritable collaboration entre administrations fiscales via l'échange spontané d'in- formations lorsque sont découverts ici ou là, sur le territoire de l'Union, des montages frauduleux reproductibles. En atteste le récent scandale des CumEx et des CumCum. Ces fraudes fiscales appuyées sur la pratique financière de l'arbitrage des dividendes ont été repérées dès 2011 en Allemagne18. Leur reproduction au Danemark aurait sans doute pu être évitée si les autorités allemandes n'avaient pas attendu deux ans pour avertir leurs homologues danoises19. Une responsabilité partagée dans le domaine de la lutte contre la fraude reste indispensable en matière d'impôts directs comme en matière de TVA, terrain sur lequel elle a commencé à prendre corps avec la création en 2010 d'EUROFISC. Même si, il convient de le rappeler, tous les États n'y participent pas encore20.

L'avenir de la coopération entre administrations n'est donc pas lié à la seule adaptation des normes d'échanges, mais dépendra de la manière dont les États s'empareront de ces outils, à charge pour l'Union européenne de leur ap- porter l'assistance technique nécessaire à lever leur résistance, via le programme « FISCALIS » notamment.

\section{B. L'adoption de standards communs pour la lutte contre l'évasion fiscale des entreprises}

Au-delà de la mise en place de réseaux d'échange d'informations, l'ensemble des directives adoptées en 
matière de fiscalité directe avaient jusqu'alors pour finalité de régler les situations de doubles impositions internationales les plus préjudiciables à l'achèvement du marché intérieur. Or, avec les directives ATAD établissant des règles pour lutter contre les pratiques d'évasion fiscale qui ont une incidence directe sur le fonctionnement du marché intérieur, les États se sont mis d'accord pour adopter un cadre commun de lutte contre les pratiques les plus couramment mises en œuvre par les entreprises. Le succès est indéniable mais, là encore, si les États membres ont accepté de s'engager, ce n'est pas sans lien avec les avancées réalisées sur ces questions dans le cadre de l'OCDE. L'échec de la taxe sur les activités numériques en est le parfait contre-exemple, aucune solution européenne n'ayant pu être dégagée avant que n'émerge un consensus international.

En tout état de cause, les directives ATAD imposent désormais aux États membres d'introduire dans leurs législations nationales des règles destinées à garantir un " niveau minimal de protection des systèmes nationaux d'imposition des sociétés contre les pratiques d'évasion fiscale ». Ainsi, la directive ATAD 1 du 12 juillet 201621 pré- voit la mise en place d'une imposition à la sortie, une exit tax, sur les plus-values latentes réalisées par les entreprises qui transfèrent des actifs ou leur résidence fiscale. Elle impose également une règlementation SEC (sociétés étrangères contrôlées) destinée à décourager la délocalisation de bénéfices par la création d'entités établies dans des pays à fiscalité faible ou nulle. Des dispositions ciblant les dispositifs dits hybrides sont en outre rendues obligatoires afin de répondre aux situations dans lesquelles des divergences de qualification juridique d'un même contribuable ou d'un même paiement entre les États sont exploitées pour obtenir une réduction de la charge fiscale résultant d'une double déduction, d'une déduction sans inclusion ou d'une double non-imposition. Cela vaut également pour les situations transfrontières impliquant des établissements stables ou des entités résidentes d'État tiers depuis l'adoption de la directive ATAD 2 du 29 mai 201722. L'arsenal rendu obligatoire com- prend également d'une part une règle de limitation des intérêts déductibles des résultats imposables des entreprises, afin d'éviter que celles-ci ne réduisent leur résultat imposable par le paie- ment et la déduction d'intérêts excessifs 23 , et d'autre part l'introduction d'une clause anti-abus générale en matière d'impôt sur les sociétés.

Les efforts consentis sont a priori conséquents, et le panel de dispositions visant la planification fiscale agressive parait impressionnant. En les adoptant, les États semblent avoir admis que la résorption de ces comportements est une condition sine qua non d'une concurrence fiscale non biaisée en Europe. Pour autant, là encore, l'enthousiasme doit être nuancé. Certaines voies essentielles d'optimisation fiscale agressive restent ouvertes. Ainsi, la proposition de conditionner la circulation intra-UE des dividendes et intérêts au paiement d'une retenue à la source lors de leur distribution hors Union européenne n'a pas été discutée, alors même qu'elle est considérée par certain «comme indispensable pour annuler tout intérêt aux pratiques de chalandage fiscal au sein de l'Union $» 24$. Or, subsistent encore au sein de l'UE des États-tunnels comme en attestent les recommandations 2019 de la Commission au titre du semestre européen. Le Luxembourg, l'Irlande mais aussi Malte ont ainsi été alertés sur la nécessité de prêter attention à l'exposition de leurs systèmes fiscaux à la planification fiscale agressive « en se concentrant en particulier sur les paiements sortants »25. Cette situation interpelle; d'autant que, si les États ont réussi à se mettre d'accord en décembre 2017 sur le principe d'une « liste noire » européenne des pays et territoires non coopératifs élaborée par le groupe du « code de conduite », aucun accord n'a à ce jour pu être trouvé sur les contre-mesures fiscales à adopter. Par ailleurs, la directive ATAD 1 laisse une marge de manœuvre non négligeable aux États au stade de sa transposition qui permettra aux plus rétifs d'en limiter l'impact. S'agissant par exemple de la clause SEC qui vise à permettre à un État de taxer au nom d'une mère résidente les bénéfices non versés d'une filiale étrangère contrôlée située dans un État à fiscalité privilégiée, la directive propose deux approches différentes : une approche par entité, qualifiée de « hard», qui permet d'inclure tous les revenus passifs de la société étrangère, et une approche transactionnelle, dite «soft », qui ne permet de taxer que les revenus non distribués qui proviennent d'un montage non authentique mis en place dans le but d'obtenir un avantage fiscal. Ici le forum shopping ne sera donc pas totalement supprimé. Ainsi, la Belgique, mais aussi le Luxembourg, terres d'accueil de holdings et initialement op- posées à l'introduction d'un tel dispositif dans leur législation, ont choisi cette seconde option26, Dans le même temps le Parlement européen déplorait 
l'existence de ces deux approches et exhortait les États à opter pour la première27. Par ailleurs, l'introduction de ces mesures dans les législations nationales ne manquera de poser d'épineuses questions juridiques : des interrogations sur leur coordination avec de potentiels dispositifs nationaux équivalents ou concurrents, mais également avec les obligations conventionnelles des États concernés. Paradoxalement, il n'est exclu pas que certains dispositifs de la directive ATAD ne soient pas contraires au droit primaire, notamment les libertés de circulation28, alors même que l'une des justifications à l'élaboration de ce « cadre commun » a justement été, selon les termes même de la directive, d'apporter aux contribuables de la sécurité juridique « dans le sens où ces mesures seraient compatibles avec le droit de l'Union »29.

Finalement l'apport majeur de ces textes ne sera peut-être pas ce qu'il paraît être à première vue. Si quelques États ne disposaient pas encore de toute la panoplie de contre-mesures visées par les directives ATAD30, la plupart d'entre eux s'en étaient déjà dotés. Ainsi, pour exemple, la France disposait déjà d'un régime SEC, d'un mécanisme de limitation de déduction des charges financières, d'une clause anti-abus générale et même d'un dispositif anti-hybride. Plus significativement, l'adoption de cette directive pourrait être la voie d'entrée pour un contrôle européen approfondi des politiques nationales de lutte contre les comportement abusifs et frauduleux.

\section{... doublée d'un contrôle européen grandissant des modalités de leur mise en œuvre par les États membres}

Améliorer les instruments étatiques de lutte contre les comportements frauduleux et abusifs ne suffit pas. Encore faut-il, pour éviter les dis- torsions de concurrence et assurer la discipline budgétaire macroéconomique au sein de l'UE, que les administrations nationales, seules compétentes en la matière, en usent. Or, jusqu'à récemment, les seules mesures de contrainte sur les États membres reposaient, non sans succès d'ailleurs, sur l'examen des pairs et la vigilance des médias. Cette situation pourrait néanmoins prendre fin dès lors que le droit de l'Union semble aujourd'hui faire peser sur les États membres une obligation de lutter contre les comportements frauduleux ou abusifs, qu'ils mettent en cause des règles européennes ou des règles nationales.

\section{A. L'obligation de lutter contre les abus de normes fiscales européennes}

Dans les domaines harmonisés, la fraude ou l'évasion fiscale peuvent être encouragées par les États. Lorsqu'elle est rendue possible par des normes nationales non conformes au droit de l'Union, l'État concerné encourt une sanction. À titre d'exemple, en matière de TVA, à la suite des « Malta files », la Commission européenne a décidé de lancer, le 8 mars 2018, une procédure d'infraction contre Chypre, la Grèce et Malte à propos du traitement fiscal favorable accordé par ces pays aux acquisitions de yachts privés 31 , le traitement en cause résultant d'une « mauvaise application » des dispositions de la directive 2006/11232. Lorsqu'est en cause non pas la législation nationale, mais le comportement du contribuable, la Cour de justice rappelle « que la lutte contre la fraude, l'évasion fiscale et les abus éventuels est un objectif reconnu et encouragé par la directive 2006/112 ». Elle en déduit que les administrations nationales sont, en matière de TVA, habilitées à remettre en cause des pratiques frauduleuses ou abusives, y compris en l'absence de dispositions du droit national les y autorisant, dès lors que leur interdiction résulte d'un principe général du droit de l'Union33. Plus encore, certaines décisions précisent très clairement que « le droit de l'Union exige des autorités et des juridictions nationales qu'elles refusent le bénéfice du droit à déduction s'il est établi, au vu d'éléments objectifs, que ce droit est invoqué frauduleusement ou abusivement34 » et instaurent une véritable obligation à la charge des États membres. Par ailleurs, nonobstant l'autonomie procédurale des États, la Cour a, dans la décision Taricco, jugé sur le fondement de l'article 325 du TFUE35 et de la convention relative à la protection de intérêts financiers de 1'Union du 26 juillet 1995, que «des sanctions pénales peuvent cependant être indispensables pour combattre de manière effective et dissuasive certains cas de fraude grave à la TVA »36. Contre l'avis du Conseil qui contestait que la TVA entre dans le 
champ d'application de ladite convention37, la Cour a considéré que « les États membres doivent prendre les mesures nécessaires pour que les comportements constitutifs d'une fraude portant atteinte aux intérêts financiers de l'Union soient passibles de sanctions pénales effectives, proportionnées et dissuasives, incluant, au moins dans le cas de fraude grave, des peines privatives de liberté ». En conséquence, il a été ordonné au juge italien de « laisser inappliquées des règles nationales de prescription faisant obstacle à l'infliction de sanctions pénales effectives et dissuasives dans un nombre considérable de cas de fraudes graves portant atteinte aux intérêts financiers de l'Union européenne »38. Certains changements législatifs récents devraient en outre conduire à l'intensification du contrôle de la Cour sur les politiques étatiques de lutte contre les comportement frauduleux et abusifs en matière de TVA dans les an- nées à venir. En effet, la directive (UE) 2017/1371 du 5 juillet 2017 relative à la lutte contre la fraude portant atteinte aux intérêts financiers de l'Union au moyen du droit pénal prévoit désormais expressément, malgré l'opposition initiale de certains États39, que les fraudes les plus graves à la TVA, c'est-à-dire celles qui ont un lien avec le territoire de plusieurs États et entraînent un préjudice d'au moins dix millions d'euros re- lèvent de la catégorie des infractions aux intérêts financiers de l'Union. Si le texte ne définit pas, les concernant, un régime commun de sanctions et n'exclut donc pas totalement les risques de forum shopping40, il impose néanmoins aux États membres d'établir des sanctions pénales incluant une peine d'emprisonnent maximale d'au moins quatre ans et de prévoir des mesures pour que «l'enquête, les poursuites, le jugement et la décision judiciaire sur les infractions pénales puissent intervenir pendant une période d'au moins cinq ans après que ces infractions pénales ont été commises ». En outre, s'agissant des États ayant pris part à la coopération renforcée pour la mise en œuvre du parquet européen41, l'intervention du Procureur européen pourra dans une certaine mesure suppléer à l'inaction potentielle par des autorités nationales 42 .

Même si l'européisation des dispositifs de lutte contre la fraude fiscale semble circonscrite à la TVA, seul impôt national pour lequel un lien avec le budget européen a pu être sollicité43, cela ne signifie pas pour autant que les États sont totalement libres de définir l'intensité de leur action lorsque des contribuables fraudent ou abusent de règles harmonisées en matière de fiscalité directe. À ce propos, la Cour de justice a récemment admis, contre l'avis de son Avocat général, que le principe général selon lequel les justiciables ne sauraient frauduleusement ou abusivement se prévaloir des normes du droit de l'Union peut être invoqué pour refuser à un contribuable le bénéfice d'un avantage fiscal prévu par une directive (en l'espèce, la directive 2003/49/CE du 3 juin 2003 concernant un régime fiscal commun applicable aux paiements d'intérêts et re- devances), alors même que l'État concerné ne disposerait dans sa législation interne d'aucun dispositif anti-abus de droit. La possibilité de $r$ fuser l'accès à un avantage fiscal prévu par une directive en présence d'un abus ou d'une fraude a donc été admise. Pourtant, le droit primaire de l'Union ne prévoit, s'agissant des impôts directs des entreprises, aucune disposition équivalente à celle de l'article 325, 1 et 2 du Traité sur le fonctionnement de l'Union européenne permettant d'ancrer textuellement une obligation de perception effective de ces prélèvements à la charge des États44. Or, pour la Cour il semble qu'une telle obligation existe bien puisqu'elle affirme « qu'un État membre doit refuser le bénéfice des dispositions du droit de l'Union lorsque celles-ci sont invoquées non pas en vue de réaliser les objectifs de ces dispositions, mais dans le but de bénéficier d'un avantage du droit de l'Union alors que les conditions pour bénéficier de cet avantage ne sont que formellement remplies ». La formulation retenue ici est impérative : la répression des fraudes et abus aux dispositions de directives, en matière de fiscalité directe, n'apparaît donc pas comme une simple faculté laissée aux États membres mais comme un véritable devoir45.

On peut donc penser que la Cour considérerait que les États qui laissent prospérer des abus au droit de l'Union, que ce soit en matière de TVA ou en matière d'impositions directes, agissent en contradiction avec le principe général du droit de l'Union interdisant les pratiques abusives. Et dès lors qu'un « manquement peut découler de l'existence d'une pratique administrative qui viole ce droit (de l'Union) lorsqu'elle présente un certain degré de constance et de généralité » 46 , il n'est pas interdit de penser que les États défaillants puissent sur ce fondement faire l'objet de sanctions. Si la question se pose différemment s'agissant des abus perpétrés contre des dispositions fiscales nationales non harmonisées, la généralisation via la directive ATAD 1 d'une clause anti-abus générale en matière d'imposition des sociétés devrait ouvrir 
la voie à un contrôle européen de l'action des États en la matière.

$B$.

Une obligation en devenir hors du champ d'application du droit de l'Union

Le point de savoir si la lutte des États membres contre les fraudes ou abus menés contre leurs propres législations fiscales constitue une obligation pour ces derniers est une question à la- quelle la Cour de Justice a pour l'instant semblé répondre par la négative. Ainsi dans l'arrêt $3 \mathrm{M}$. Italia, elle a affirmé qu' "il n'existe en droit fiscal de l'Union aucun principe général duquel découlerait une obligation pour les États membres de lutter contre les pratiques abusives dans le domaine de la fiscalité directe... lorsque l'opération procède de telles pratiques et que le droit de l'Union n'est pas en jeu »47. Bien que la tolérance envers les comportements abusifs et frauduleux puisse être un moyen de la concurrence fiscale dommageable entre États, il n'est donc pas cer- tain que l'effectivité de la lutte menée au plan national puisse être contrôlée sur le fondement du principe général d'interdiction des pratiques abusives 48 .

Pour autant, cet état du droit n'a pas suffi, comme chacun le sait, à décourager l'activisme de la Commission européenne. Elle s'est d'abord emparée de la règlementation des aides d'État pour remettre en cause les décisions anticipatives, accords préalables sur prix de transfert et autres rulings par lesquels les États peuvent contribuer à la stratégie de fraude ou d'optimisation fiscale agressive d'une entreprise, en renonçant sélectivement à prélever l'impôt national. Elle considère en effet, sous le contrôle de la Cour de justice, qu'est prohibé sur le fondement de l'article $107 \mathrm{du}$ TFUE, tout rescrit qui « applique erronément le droit fiscal national »49 et induit un résultat d'imposition moins élevé. Allant encore plus loin encore, elle postule que les accords préalables de prix de transfert dé- terminant le traitement fiscal d'opérations intragroupes doivent être présumés constitutifs d'une aide d'État lorsque l'autorité fiscale qui les délivre accepte un prix de transfert qui ne respecte pas le principe de pleine concurrence, que cet État « ait ou non repris ce principe dans son régime national »50. Le principe de pleine concurrence ferait « nécessairement partie intégrante de l'examen, au titre de l'article 107, para- graphe 1, TFUE, des mesures fiscales accordées aux sociétés d'un groupe ». Dénoncée par les États membres comme relevant d'une " harmonisation déguisée », cette analyse, qui conduit à imposer aux États membres une règle d'assiette déconnectée des prescriptions de leur droit national et du droit conventionnel, a pour l'instant été validée par la juridiction européenne51. Dans d'autres hypothèses, et de manière encore plus subtile, le raisonnement par lequel la Commission identifie une aide d'État repose non pas sur une prétendue application erronée du droit fiscal matériel national, mais sur le fait que les autorités nationales se seraient abstenues de sanctionner un comportement abusif alors que la législation nationale en matière de procédures fiscales le leur permettait. Pour l'identification des aides d'État, les dispositifs anti-abus qui reposent sur une analyse du comportement du contribuable sont assimilés à des règles d'assiette et intégrés au régime fiscal de référence. À ce titre, la Commission a par exemple ordonné la récupération d'une aide fiscale octroyée par le Luxembourg au groupe ENGIE au moyen de décisions fiscales anticipatives. Dans ce dossier, bien qu'à titre subsidiaire, la Commission suggère que l'avantage sélectif consenti à ENGIE pourrait résulter de la non mise en œuvre des dispositions fiscales luxembourgeoises sur l'abus de droit qui, selon la Commission, « font partie du système de référence, puisqu'elles assurent la cohérence interne du système fiscal de référence luxembourgeois »52. Dans le même ordre d'idées, mais de manière différente puisqu'était ici en cause un régime d'aides et non une aide individuelle, la Commission a considéré qu'une disposition britannique mettant certaines entreprises multinationales à l'abri du régime des sociétés étrangères contrôlées au titre de revenus de financement intra-groupe constitue une aide d'État53. Quelle que soit l'analyse que fera la Cour de justice de l'argumentation de la Com- mission, ces deux affaires attestent de sa volonté de contraindre, avec les moyens juridiques que lui offre le droit de l'Union, les États membres à plus de loyauté dans la mise en œuvre de leurs politiques nationales de lutte contre la fraude et l'évasion fiscales. Mais, la procédure utilisée ici est fragile. Politiquement d'abord, elle cristallise le rejet des États qui persistent à soutenir que le droit de la concurrence est dévoyé de ses objec- tifs initiaux, la Commission empiétant sur leurs compétences en matière de fiscalité directe54; juridiquement ensuite, car sauf à considérer, comme pour le principe de libre 
concurrence, qu'il existe pour le contrôle des aides d'État un principe obligeant les États membres à lutter contre les abus de leurs législations nationales, l'identification d'un avantage fiscal consistant en la nonrépression d'un tel abus dépend de l'existence d'un dispositif interdisant les pratiques abusives dans le cadre fiscal de référence, à sa- voir le droit national. L'abandon de la procédure menée contre le Luxembourg pour octroi d'une aide d'État en faveur de la société McDonald's en est d'ailleurs la parfaite illustration. Alors même que la Commission avait constaté l'existence d'une double non-imposition, aux États-Unis et au Luxembourg, de certains des bénéfices de la succursale américaine de la société luxembourgeoise McD Europe Franchising, elle a du conclure, en l'absence de dispositif anti-hybride dans la législation luxembourgeoise, que cet avantage, non conforme au principe d'équité fis- cale, résultait uniquement d'une incompatibilité entre les législations fiscales luxembourgeoises et américaines et non d'une application erronée de la convention liant le Luxembourg et les États- Unis .55

Ceci étant dit, indépendamment de la reconnaissance d'un principe général imposant aux États de lutter contre les comportements abusifs ou frauduleux en matière de fiscalité directe, l'entrée en vigueur des directives ATAD pourrait dans l'avenir donner prise à une sécurisation du contrôle de la Commission : désormais, les dis- positifs anti-abus visés par ces textes devront nécessairement être introduits en droit interne et s'intégreront au système de référence pour l'identification d'aides d'État prohibées. Parmi ces dispositifs, la clause anti-abus générale de l'article 6 de la directive ATAD qui permet aux administrations fiscales d'écarter les montages ou séries de montages non authentiques « mis en place pour obtenir, à titre d'objectif principal ou au titre d'un des objectifs principaux un avantage fiscal allant à l'encontre de l'objet du droit fiscal applicable » offrira à la Commission l'occasion d'une extension sensible du champ de son contrôle. «Première intervention d'une directive en matière de fiscalité nationale des sociétés puisqu'elle s'applique, [aux situations transfrontières ] mais également aux situations purement internes »56, elle pourrait en effet être la base lé- gale sur laquelle la Commission contrôlera l'effectivité de la lutte des États membres contre les comportements abusifs des entreprises au moins en matière de fiscalité directe de leur bénéfice57, y compris lorsque la règle abusée n'est pas issue du droit de l'Union. La notion même de pratiques abusives sera européanisée puisque les autorités nationales devraient perdre toute autonomie dans sa définition, en raison du contrôle que le juge communautaire sera fondé à pratiquer58. En effet, le juge national devra désormais, en cas de doute sérieux, se tourner vers la CJUE pour décider si tels ou tels montages, y compris en situation strictement interne, constituent des comportements qui doivent être prohibés. Il n'est guère douteux qu'elle y trouvera un nouvel « instrument de politique » jurisprudentielle qui pourra être mis au service de «l'intégration fiscale » européenne.

Bien que les comportements d'évitement d'un certain nombre de contribuables apparaissent comme la première cible des textes récemment adoptés, l'évolution récente du droit de l'Union s'adresse tout aussi clairement aux États. Il s'agit en réalité de faciliter - au besoin même de forcer - l'émergence d'une véritable confiance mutuelle des administrations fiscales nationales. Car, qu'il s'agisse, comme c'est le cas aujourd'hui, d'assurer la coexistence pacifiée des libertés de circulation au sein du marché intérieur avec la persistance de systèmes fiscaux nationaux, ou, peut- être, plus tard d'appliquer des règles fiscales harmonisées, il est évident, même si ces aspects sont trop peu souvent soulignés, que cela ne peut se faire sans le concours loyal de chacune d'elles59.

\section{Notes de bas de page}

1 J.- Cl. Juncker, Orientations politiques pour la prochaine Commission européenne, Discours d'ouverture de la session plénière du Parlement européen, juil. 2014.

2. Proposition de directive modifiant la directive 2006/112/CE en ce qui concerne l'introduction de mesures techniques détaillées pour le fonctionnement du système de TVA définitif pour la taxation des échanges entre les États membres, 25.5.2018, COM (2018) 329 
final. La fraude à la TVA aurait représenté 137 milliards en 2017 : Study and Reports on the VAT GAP in the EU-28 Member States : 2019 Final Report, TAXUD/2015/CC/132, 4 septembre 2019.

3 E. Dufau et P.-J. Douvier, Optimisation, évasion et fraude fiscales : le nécessaire maintien de frontières au tracé délicat : Rev. Droit \& affaires 2015, n¹2, §18.

4 Commission européenne, Communication sur la transparence fiscale pour lutter contre la fraude et l'évasion fiscales, 18 mars 2015, COM (2015) 136 final.

5 Sur une proposition pour la clarification des notions de fraude, d'évasion et

d'optimisation fiscale : voir B. Peyrol, J.-F. Parigi, Rapport d'information relatif à l'évasion fiscale internationale des entreprises, 12 sept. 2018, AN, Commission des finances, de l'économie générale et du contrôle budgétaire, $\mathrm{n}^{\circ} 1236$.

6 Voir pour un exemple récent, la condamnation du régime français de retenue à la source sur les dividendes versés hors de France : CJUE, 5e ch, 22 nov 2018, aff. C-575/17, Sofina SA, Rebelco SA, Sidro SA, pt 77 : la Cour juge que la retenue à la source perçue sur les dividendes versés à des sociétés non résidentes déficitaires ne peut être justifiée par l'efficacité du recouvrement de l'impôt.

7 Proposition de directive du Parlement européen et du Conseil relative à la lutte contre la fraude portant atteinte aux intérêts financiers de l'Union au moyen du droit pénal, 11 juil. 2012, COM

(2012) 363 final, p.4.

8 Directive 2014/107 du Conseil du 9 décembre 2014 modifiant la directive 2011/16/UE en ce qui concerne l'échange automatique et obligatoire d'informations dans le domaine fiscal : JOUE L359, 16 déc. 2014, p.1

9 Directive (UE) 2016/2258 du Conseil du 6 décembre 2016 modifiant la directive 2011/16/UE en ce qui concerne l'accès des autorités fiscales aux informations relatives à la lutte contre le blanchiment de capitaux : JOUE L342, 16 déc. 2016, p.1.

10 Directive (UE) 2015/2376 du Conseil du 8 décembre 2015 modifiant la directive 2011/16/UE en ce qui concerne l'échange automatique et obligatoire d'informations dans le domaine fiscal : JOUE L 332, 18 déc. 2015, p.1.

11 Directive (UE) 2016/881 du Conseil du 25 mai 2016 modifiant la directive 2011/16/UE en ce qui concerne l'échange automatique et obligatoire d'informations dans le domaine fiscal : JOUE L146, 3 juin 2016, p.8.

12 Dr. fisc. $2016, \mathrm{n}^{\circ} 23$, act. 355.

13 Directive (UE) 2018/822 du Conseil du 25 mai 2018 modifiant la directive 2011/16/UE en ce qui concerne l'échange automatique et obligatoire d'informations dans le domaine fiscal en rapport avec les dispositifs transfrontières devant faire l'objet d'une déclaration : JOUE L 139, 5 juin 2018, p.1. Cette dernière devait être transposée avant le 31 décembre 2019 avec dépôt des premières déclarations pour le ler aout 2020.

14 M.-H. Pinard - Fabro, Optimisation fiscale transfrontière : de nouvelles obligations dès à présent : FR Lefebvre 2018, $\mathrm{n}^{\circ} 30,5$.

15 Commission européenne, Aperçu et évaluation des statistiques et des informations relatives aux échanges automatiques dans le domaine de la fiscalité directe, 17 déc. 2018, COM (2018) 844 final ; voir également Evaluation of the Council Directive 2011/16/UE on administrative coopération field of taxation and repealing Directive 77/799/EEC, 12 oct. 2019, SWD (2019) 327 final.

16 Rapport de la Commission européenne au Parlement européen, au conseil, au comité économique et social européen et au comité des régions, Programmes de citoyenneté et de résidence par investissement dans l'Union européenne, 13 janvier 2019, (COM(2019) 12 final : sur les risques de fraude fiscale, p. 18 et s.

17 P. Moscovici, Report on financial crimes, tax evasion and tax avoidance, débats, 25 mars 2019.

18 A. Michel, M. Vaudano et J. Baruch, «Cum ExFiles » : l'histoire secrète du casse du siècle : le Monde, 18 octobre 2018.

19 A. Michel, M. Vaudano et J. Baruch, «CumExFiles » : la coopération fiscale européenne, en chantier, a connu des ratés ; Le Monde, 18 octobre 2018.

20 Le Parlement européen rappelle que la participation à EUROFISC n'est pas obligatoire et propose que cette participation conditionne l'obtention de fonds européens : rapport sur la criminalité financière, la 
fraude fiscale et l'évasion fiscale, Parlement européen, 26 mars 2019, P8_TA-PRO (2019)0240, § 164.

21 Directive (UE) 2016/1164 du Conseil du 12 juillet 2016 établissant des règles pour lutter contre les pratiques d'évasion fiscale qui ont une incidence directe sur le fonctionnement du marché intérieur ; JOUE L193, 19 juil. 2016, p.1. 22 Directive (UE) 2017/952 du Conseil du 29 mai 2017 modifiant la directive (UE) 2016/1164 en ce qui concerne les dispositifs hybrides faisant intervenir des pays tiers ; JOUE L 144, 7 juin 2017, p.1 ; Dr. fisc. 27/2017, comm. 386.

$23 \mathrm{La}$ transposition est obligatoire, sauf pour la règle de limitation des intérêts. Il est en effet possible d'en reporter la transposition au 1er janvier 2024 si les règles de limitation de la déductibilité des charges financières nationales sont jugées aussi efficaces que la règle ATAD par la Commission européenne (Directive (UE) 2016/1164, art. 11).

24 Conseil des prélèvements obligatoires, Adapter l'Impôt sur les sociétés à une économie ouverte, décembre 2016, p. 141 et s. ;

B. Lignereux, Le principe de territorialité de l'IS, Rapport Particulier n 4, CPO, 2016,p. 106 et s.

25 Une étude publiée sur la plateforme concernant la bonne gouvernance dans le domaine fiscal affirme d'ailleurs que les «États-conduits les plus importants sont des États européens »: A. Lejour, CPB et Tilburg University, The (un)fairness of corporate taxation, Déc 2018, diaporama 15.

26 O. Herman, P. Lacroix, Du changement dans les règles relatives aux sociétés étrangères contrôlées, L'écho, 5 février 2019 ; C. Beernaerts, J. Meeus et I. Kam, Fiscalité internationale - Benelux : Chronique de l'année 2018 ; Dr. fisc. 2019, n 10, 192.

27 Rapport sur la criminalité financière, la fraude fiscale et l'évasion fiscale, Parlement européen, 26 mars 2019, P8_TA-PRO (2019)0240, § 46.

28 Voir sur ce point A. Maîtrot de la Motte, Les causes anti- abus et le droit de l'Union européenne ; Dr. fisc. 2016, $\mathrm{n}^{\circ} 13$, comm. 257.

29 La question de la compatibilité de la clause anti-abus générale applicable en matière d'IS avec la liberté d'établissement pourrait néanmoins être réglée. La Cour de justice a en effet considéré que le principe d'interdiction des pratiques abusives « constitue un général du droit de l'Union qui s'applique indépendamment du point de savoir si les droits et les avantages dont il est abusé trouvent leur fondement dans les traités, dans un règlement ou une directive »: CJUE, gr.ch., 26 févr. 2019, aff. C-115/16, n. Luxembourg 1 et a., pts 96 et 101. Par-delà les différences de formulation existant entre les directives et la jurisprudence de la CJUE, «c'est bien un concept unique d'abus auquel il convient de se référer » : Nicolas de Boynes, La CJUE donne son éclairage sur la notion d'abus de droit : Dr. fisc. 2019, ${ }^{\circ} 21$, comm. 275). 30 Voir pour un état des lieux en 2017, Fiche Thématique du semestre européen, Lutter contre la planification fiscale agressive, 20 novembre 2017.

31 Communiqué de presse, 8 mars 2018, IP/18/1451

32 De son côté, l'administration fiscale française a été contrainte de remettre en cause la réfaction forfaitaire admise pour déterminer la part des loyers tirés de la location de navires imposable en France (BOI-TVACHAMP-20-50-30, §40).

33 CJUE, 4e ch., 22 nov. 2017, aff. C-252/16, E. Cussens.e.a. c/T.G. Brosnan, pt. 31 ; et pour des décisions antérieures CJUE, 18 déc. 2014, C-131/13, C-6163/13 et 164/13, Schoenimport « Italmoda » Mariano Oréviti vof, pt.44.

34 CJUE, 13 fév. 2014, aff. C-18/13, Maks Pen, pts. 33 à 39. ; CJUE, C-617/10, 26 fév. 2013, Aklagaren c/ Åkerberg Fransson, point 25 : « chaque État membre a l'obligation de prendre toutes les mesures législatives et administratives propres à garantir la perception de l'intégralité de la TVA due sur son territoire et à lutter contre la fraude ».

35 TFUE, art. 325 : «L’Union et les États membres combattent la fraude et tout autre activité illégale portant atteinte aux intérêts financiers de l'Union par des mesures prises conformément au présent article qui sont dissuasives et offrent une protection effective dans les États membres, ainsi que dans les institutions, organes et organismes de l'Union $\gg$.

36 CJUE, grde ch., 8 sept. 2015, aff. C-105/14, Taricco, p. 39 et 40.

37 D. Berlin, La lutte contre la fraude : action et politique de l'Union ?, in La fraude et le droit de l'Union européenne, Bruylant, 2017, p.42, note 12. 
38 Sous réserve cependant du respect du principe de la légalité des peines et délits ; CJUE, grde ch., 5 déc. 2017, aff. C-42/17, M. A.S. et M. B, pt. 41.

39 Voir sur ce point, A. Turmo, Le Parquet européen et la lutte contre la fraude ; Beaucoup de bruit pour rien, in La fraude et le droit de l'Union européenne, Bruylant, 2017, p. 127 et s.

40 C. Di Francesco Maesa, Vers un Parquet européen : entre effectivité et droits fondamentaux, Thèse Strasbourg, 2017, p. 80.

41 Règlement (UE) 2017/1939 du 12 octobre 2017 mettant en œuvre une coopération renforcée concernant la création du Parquet européen.

42 G. Duteil, J.-F. Thony, Le parquet européen et la lutte contre les fraudes à la TVA : AJ Pénal 2018, p. 290.

43 Le Parquet européen n'a aucune compétence pour décider de poursuites contre les infractions pénales connexes à la fraude à la TVA portant sur les impôts directs. Elles sont expressément exclues de son champ de compétence, alors même qu'en principe le Parquet européen est habilité à poursuivre les « infractions pénales indissociablement liées aux infractions « PIF ».

44 Conclusions Kokott, 1er mars 2018, Affaire C-115/16, N Luxembourg 1 contre Skatteministeriet, pt. 98 et $\mathrm{s}$ : au soutien de la non application du principe général d'interdiction des pratiques abusives en matière de fiscalité directe, l'Avocat général faisait valoir que « le droit de l'Union impose aux États membre une obligation de perception (effective) de la TVA conformément à l'article 325, paragraphes 1 et 2, TFUE alors que ce n'est pas le cas en matière de fiscalité directe sur les revenus $»$.

45 V. BASSANI, L'interdiction de l'abus de droit : principe général de l'Union ? - . - A propos des arrêts rendus par la grande chambre de la Cour de Justice du 26 février 2019 : Europe 2019, n 4, Etude 3.

46 CJCE, 10 sept. 2009, aff. C-416/07, Commission c/ Grèce, pt. 24 ; CJUE, 12 mai 2005, aff. C-278/03, Commission c/ république Italienne, pt.13

47 CJCE, 29 mars 2012, C-417-10, 3M Italia,, pt. 32.

48 Voir sur l'incertitude du champ d'application du principe général du droit d'interdiction des pratiques abusives, D. Simon, Portée en droit national, Europe 2012, n 12 , comm. 177.

49 Communication de la Commission relative à la notion d'aide d'État visée à l'article 107, paraphe 1, du Traite sur le fonctionnement de l'UE, 2016/C 262/01, §174.

50 Comm. UE, déc. 2017/502, 21 oct. 2015 concernant l'aide d'État SA.38374 (2014/C ex 2014/NN) mise à exécution par les Pays-Bas en faveur de Starbucks, pt. 264 ; Comm. UE, déc. n 2016/2326, 21 oct. 2015 concernant l'aide d'État SA.38375 (2014/C ex 2014/NN) mise à exécution par le Luxembourg en faveur de Fiat, pt 228.

51 Tr. UE, 24 sept. 2019 ; Royaume des Pays-Bas, Starbucks Corp c/ Comm., aff. T-760/15 et T-636/16, pt. 162

52 Commission européenne concernant l'aide d'État SA 44888 (2016/C) mise à exécution par le Luxembourg, faveur d'ENGIE, C (2018) 3839 final du 20 juin 2018, § 289 et suivants. O. Peiffert, Premiers arrêts du Tribunal de l'UE en matière de rescrits fiscaux : la Commission européenne perd un set mais remporte le match : Dr. fisc. 2019, n 40, act. 419.

53 Commission européenne, 2 avr. 2019, IP/19/1948.

54 Juridiquement, l'action en matière de fiscalité directe sur le fondement de la règlementation des aides d'État ne fait plus débat. Le Tribunal de l'UE vient de le rappeler que « les interventions des États

membres dans les domaines qui n'ont pas fait l'objet d'une harmonisation dans l'Union, tels que la fiscalité directe, ne sont pas exclus du champ d'application de la réglementation relative au contrôle des aides d'État »; Trib. UE, 7e ch., 14 février 2019, aff. T-131- 1/16 à T-263/16, Belgique/ Commission.

55 Commission européenne, décision UE 2019/1252, 19 sept. 2018 relative aux

décisions fiscales anticipatives SA. 38945 (2015/C) accordées par le Luxembourg à McDonald's Europe ; JOUE L.195/23 du 23 juil. 2019.

56 J. Turot, La liberté de gestion des entreprises entre enfer et paradis (et plus près de l'enfer) : Dr. fisc. 27/2017, c. 378 .

57 D. de Charette, The Anti Avoidance Directive Général Anti-Abuse Rule : A legal Basis for the Duty of 
Member states to fight tax abuse in UE Corporate Direct tax Law, EC Tax Review, Août 2019, Volume 28, $\mathrm{n}^{\circ} 4$.

58 N. de Boynes, La CJUE donne son éclairage sur la notion d'abus de droit : Dr. fisc. 2019, ${ }^{\circ} 21$, comm. 275.

59 Voir par exemple sur l'importance des aspects déclaratifs et des modalités de contrôle dans le projet ACCIS : E. de Crouy-Chanel, Le volet procédural de la proposition de directive ACCIS : naissance d'un droit européen des procédures fiscales ou mort de la proposition de directive ? : Dr. fisc. 2012, n 47 , c. 578. 